\title{
Probing the Weinberg operator at colliders
}

\author{
Benjamin Fuks $\odot,{ }^{1,2, *}$ Jonas Neundorf $\odot,{ }^{3, \dagger}$ Krisztian Peters, ${ }^{3, *}$ Richard Ruiz $\odot,{ }^{4,5,8}$ and Matthias Saimpert ${ }^{6, \|}$ \\ ${ }^{1}$ Sorbonne Université, CNRS, Laboratoire de Physique Théorique et Hautes Énergies, \\ LPTHE, F-75005 Paris, France \\ ${ }^{2}$ Institut Universitaire de France, 103 boulevard Saint-Michel, 75005 Paris, France \\ ${ }^{3}$ Deutsches Elektronen-Synchrotron, Notkestraße 85, 22607 Hamburg, Germany \\ ${ }^{4}$ Institute of Nuclear Physics, Polish Academy of Sciences, ul. Radzikowskiego, Cracow 31-342, Poland \\ ${ }^{5}$ Centre for Cosmology, Particle Physics and Phenomenology (CP3), Université Catholique de Louvain, \\ Chemin du Cyclotron, Louvain la Neuve, B-1348, Belgium \\ ${ }^{6}$ CERN-1211, Geneva 23, Switzerland
}

(Received 5 March 2021; accepted 10 May 2021; published 11 June 2021)

\begin{abstract}
Motivated by searches for $0 \nu \beta \beta$ decay in nuclear experiments and collider probes of lepton number violation at dimension $d \geq 7$, we investigate the sensitivity to the $d=5$ Weinberg operator using the nonresonant signature $p p \rightarrow \ell^{ \pm} \ell^{\prime \pm} j j$ at the LHC. We develop a prescription for the operator that is applicable in collisions and decays, and focus on the $\ell \ell^{\prime}=\mu \mu$ channel, which is beyond the reach of nuclear decays. For a Wilson coefficient $C_{5}^{\mu \mu}=1$, scales as heavy as $\Lambda \sim 8.3(11) \mathrm{TeV}$ can be probed with $\mathcal{L}=300 \mathrm{fb}^{-1}\left(3 \mathrm{ab}^{-1}\right)$. This translates to an effective $\mu \mu$ Majorana mass of $\left|m_{\mu \mu}\right| \sim 7.3(5.4) \mathrm{GeV}$ and establishes a road map for testing the Weinberg operator at accelerators.
\end{abstract}

DOI: 10.1103/PhysRevD.103.115014

\section{INTRODUCTION}

Among the most pressing mysteries shared in cosmology, nuclear, and high-energy physics is whether neutrinos are their own antiparticles [1,2]. This importance follows from Majorana neutrinos being necessary ingredients for standard leptogenesis, grand unification, as well as new gauge symmetries. Discovering that neutrinos are Majorana particles would indicate that lepton number (LN) symmetries are not conserved below the electroweak (EW) scale and demonstrate the existence of a mass-generating mechanism beyond those responsible for chiral and EW symmetry breaking (EWSB).

Motivated by this, broad, complementary approaches are taken to explore the nature of neutrinos [3-10]. A foremost probe is the search for the neutrinoless $\beta \beta$ process $(0 \nu \beta \beta)$ in decays of nuclei. This is characterized by the transition $(A, Z) \rightarrow(A, Z+2)$ and the appearance of two same-sign electrons but an absence of neutrinos in the final state.

\footnotetext{
fuks@lpthe.jussieu.fr

jonas.neundorf@desy.de

*krisztian.peters@desy.de

\$rruiz@ifj.edu.pl

"matthias.saimpert@cern.ch
}

Published by the American Physical Society under the terms of the Creative Commons Attribution 4.0 International license. Further distribution of this work must maintain attribution to the author(s) and the published article's title, journal citation, and DOI. Funded by SCOAP ${ }^{3}$.
While no discovery has been confirmed, and assuming that the decay is mediated solely by the light neutrinos observed in nature, searches place upper limits of $79-180 \mathrm{meV}$ at 90\% confidence level (C.L.) [11] on the so-called effective $\beta \beta$ Majorana mass, given by [12]

$$
\left|m_{e e}\right|=\left|\sum_{k=1}^{3} U_{e k} m_{\nu_{k}} U_{e k}\right|
$$

In this definition, $m_{\nu_{k}}$ are the mass eigenvalues of the three light neutrinos and $U_{\ell k}$ are the Pontecorvo-MakiNakagawa-Sakata (PMNS) mixing matrix elements.

From the perspective that the Standard Model (SM) of particle physics is a low-energy effective field theory (EFT), Majorana neutrino masses, and hence $\left|m_{e e}\right|$, can be generated most minimally $[13,14]$ at dimension $d=5$ from the LN-violating Weinberg operator [15],

$$
\mathcal{L}_{5}=\frac{C_{5}^{\ell \ell^{\prime}}}{\Lambda}\left[\Phi \cdot \bar{L}_{\ell}^{c}\right]\left[L_{\ell^{\prime}} \cdot \Phi\right]+\text { H.c. }
$$

Here, $\Lambda$ is the scale at which the particles responsible for $\mathrm{LN}$ violation become relevant degrees of freedom, $C_{5}^{\ell \ell^{\prime}}$ is a flavor-dependent Wilson coefficient, $L_{\ell}^{T}=\left(\nu_{\ell}, \ell\right)$ is the left-handed (LH) lepton doublet, and $\Phi$ is the SM Higgs doublet, whose vacuum expectation value (VEV) $v=$ $\sqrt{2}\langle\Phi\rangle \approx 246 \mathrm{GeV}$ generates the quantity 


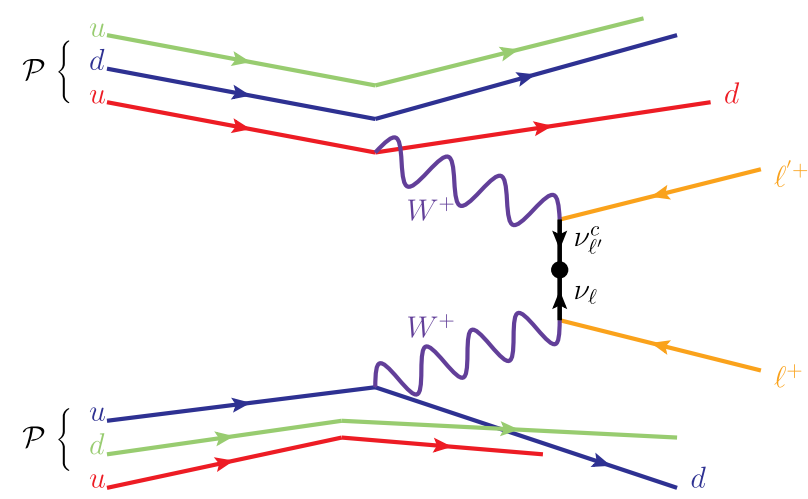

FIG. 1. Diagrammatic representation of same-sign charged lepton production through same-sign $W W$ scattering in proton collisions when mediated at dimension $d=5$.

$$
m_{\ell \ell^{\prime}}=C_{5}^{\ell \ell^{\prime}} v^{2} / \Lambda
$$

As the Weinberg operator can be realized by tree- and loop-level Seesaw models $[7,13,14]$, limits on $\left|m_{e e}\right|$ translate into lower bounds on the Seesaw scale of about $\left(\Lambda / C_{5}^{e e}\right) \gtrsim(3.3-7.6) \times 10^{14} \mathrm{GeV}$. While stringent, a caveat of this constraint is its flavor dependence. For instance, $C_{5}^{e e}$ can be zero due to a flavor symmetry [16], or be immeasurably small due to accidental cancellations [1719]. More generally, the production of same-sign leptons involving muons or taus in $(A, Z) \rightarrow(A, Z+2)$ decays is kinematically forbidden. Their production requires higher energies, implying a lack of sensitivity for nonelectron flavors of $C_{5}^{\ell \ell^{\prime}}$ at $0 \nu \beta \beta$ decay experiments.

Motivated by these limitations and by sensitivity projections for interactions at $d \geq 7$ in same-sign $W^{ \pm} W^{ \pm}$ scattering [20-23], we report an investigation into the realization of the $0 \nu \beta \beta$ process at $d=5$ in high-energy proton collisions. As shown in Fig. 1, the transition proceeds from $W^{ \pm} W^{ \pm}$scattering into same-sign charged lepton pairs $\ell^{ \pm} \ell^{\prime \pm}$ of arbitrary flavor and bridged via the coupling $m_{\ell \ell^{\prime}} \propto C_{5}^{\ell \ell} / \Lambda$. While related, this work differs from studies on the "inverse" $0 \nu \beta \beta$ process [22-25], which focus on $d \geq 7$ operators or their realizations. Moreover, this work relies on a new method for modeling the Weinberg operator that is applicable to meson and lepton decays, and establishes a road map to the Weinberg operator at accelerators. Finally, we release an implementation of this method in new and publicly available software Available from [26] for Monte Carlo (MC) simulations.

\section{THE STANDARD MODEL AT DIMENSION FIVE}

To describe Majorana neutrino masses and the $0 \nu \beta \beta$ process from $d=5$ interactions, we work in the SM effective field theory [27] and extend the SM Lagrangian $\left(\mathcal{L}_{\mathrm{SM}}\right)$ by gauge-invariant operators of $d>4$. In the canonical representation [28], the Lagrangian is given by [15]

$$
\mathcal{L}_{\mathrm{SMEFT}}=\mathcal{L}_{\mathrm{SM}}+\mathcal{L}_{5}+\mathcal{O}\left(\Lambda^{-2}\right)
$$

where $\mathcal{L}_{5}$ is defined in Eq. (2). By the power counting of Ref. [28], the Weinberg operator is the only gauge-invariant operator at $d=5$ in the SM $[15,29]$.

After EWSB, the Higgs field can be expanded about its $\mathrm{VEV}$, which in the unitary gauge reads $\sqrt{2} \Phi \approx v+h$, where $h$ is the Higgs boson. The resulting Lagrangian is

$$
\begin{aligned}
\mathcal{L}_{5}= & -\frac{C_{5}^{\ell \ell^{\prime}}}{2 \Lambda} h h \overline{\nu_{\ell}^{c}} \nu_{\ell^{\prime}}-\frac{C_{5}^{\ell \ell^{\prime}} v}{\Lambda} h \overline{\nu_{\ell}^{c}} \nu_{\ell^{\prime}} \\
& -\frac{C_{5}^{\ell \ell^{\prime}} v^{2}}{2 \Lambda} \overline{\nu_{\ell}^{c}} \nu_{\ell^{\prime}}+\text { H.c. }
\end{aligned}
$$

Here, $C_{5}^{\ell \ell^{\prime}}$ is defined in the flavor basis. The minus signs above originate from the $\mathrm{SU}(2)_{L}$-invariant product $\Phi \cdot \overline{L^{c}}=\Phi^{i} \varepsilon_{i j} \overline{L^{c j}}$, with $\varepsilon_{12}=1$. While the first two terms in Eq. (5) signify double- and single-Higgs couplings to neutrinos of flavors $\ell \ell^{\prime}$, the third term generates the $3 \times 3$ LH Majorana mass matrix $m_{\ell \ell^{\prime}}$, as defined in Eq. (3). After rotating $m_{\ell \ell^{\prime}}$ into the mass basis, the resulting eigenvalues parametrize the three neutrino mass eigenstates $m_{\nu_{k}}$ that describe neutrino oscillation data.

We make no assumption on the structure of $C_{5}^{\ell \ell^{\prime}}$. It is therefore possible under this framework that one neutrino is massless, as allowed by data [30]; that all masses scale as $m_{\nu_{k}} \sim \mathcal{O}\left(m_{\ell \ell^{\prime}}\right)$, indicating minor fine-tuning; or that $m_{\nu_{k}} \ll m_{\ell \ell^{\prime}}$, indicating strong cancellations among the $m_{\ell \ell^{\prime}}$ elements. As nuclear searches are only sensitive to $\left|m_{e e}\right|$, the latter possibilities remain underexplored.

\section{THE 0 $\nu \beta \beta$ PROCESS AT DIMENSION FIVE}

A goal of this work is to estimate the sensitivity of the Large Hadron Collider (LHC) and the high-luminosity LHC (HL-LHC) to the $0 \nu \beta \beta$ process and hence the Weinberg operator. When simulating the Weinberg operator at the LHC, difficulties arise if working in the neutrinos' mass eigenbasis. There, $d=5$ vertices are proportional to $m_{\nu_{k}}$, which are unknown and small on LHC scales, and to $U_{\ell k}$, which carry unknown phases. So while the transition in Fig. 1 may proceed through a nontrivial incoherent sum of intermediate states, individual contributions may be too small for practical computations.

We propose a solution to this complication by working in the neutrino flavor basis and treating the mass term in Eq. (5) as a "two-point vertex." From this perspective, the Weinberg operator in Fig. 1 couples one massless, LH neutrino of momentum $p$ and flavor $\ell$ with the conjugate of a second neutrino of momentum $p$ and flavor $\ell^{\prime}$. After contracting Dirac matrices, the LN-violating $\left(\nu_{\ell} \nu_{\ell^{\prime}}^{c}\right)$ current in Fig. 1 reduces to the ratio of $m_{\ell \ell^{\prime}}$ and the squared virtuality $p^{2}$. Explicitly, its graph simplifies to 


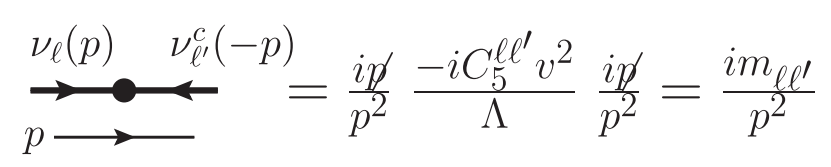

Up to corrections of $\mathcal{O}\left(\left|m_{\ell \ell^{\prime}}^{2} / p^{2}\right|\right)$, which are assumed small, one can identify the rightmost ratio as the righthanded $(\mathrm{RH})$ helicity state of an intermediate fermion with mass $m_{\ell \ell^{\prime}}$ and momentum $p$. That is, one can write

$$
\begin{gathered}
\gamma^{\alpha} P_{L} \frac{i\left(\not p+m_{\ell \ell^{\prime}}\right)}{p^{2}-m_{\ell \ell^{\prime}}^{2}} \gamma^{\beta} P_{R}=\gamma^{\alpha} P_{L} \frac{i m_{\ell \ell^{\prime}}}{p^{2}-m_{\ell \ell^{\prime}}^{2}} P_{L} \gamma^{\beta} \\
=\gamma^{\alpha} P_{L} \frac{i m_{\ell \ell^{\prime}}}{p^{2}} P_{L} \gamma^{\beta} \times\left[1+\mathcal{O}\left(\left|\frac{m_{\ell \ell^{\prime}}^{2}}{p^{2}}\right|\right)\right],
\end{gathered}
$$

where $P_{R / L}=\frac{1}{2}\left(1 \pm \gamma^{5}\right)$ are the usual chiral projection operators in four-component notation and recover the same ratio at leading power of the expansion. Intuitively, this identification follows from the inversion of helicity in LNviolating currents as discussed in Refs. [23,31-36].

As a result, up to corrections of $\mathcal{O}\left(\left|m_{\ell \ell^{\prime}}^{2} / p^{2}\right|\right)$, the $\left(\nu_{\ell} \nu_{\ell^{\prime}}^{c}\right)$ current itself can be modeled as an unphysical Majorana neutrino $N$ with mass $m_{\ell \ell^{\prime}}$ that couples to the $W$ boson and all charged leptons $\ell$ via the Lagrangian

$$
\Delta \mathcal{L}=-\frac{g_{W}}{\sqrt{2}} W_{\mu}^{+} \sum_{\ell=e}^{\tau} \bar{N} \gamma^{\mu} P_{L} \ell^{-}+\text {H.c. }
$$

Here, $g_{W} \approx 0.65$ is the $\mathrm{SU}(2)_{L}$ weak coupling constant. Up to factors of active-sterile mixing, Eq. (8) is identical to the interaction Lagrangian in the phenomenological type I seesaw model [3,37], and therefore can also be employed in LN-violating decays of hadrons and leptons.

\section{SIGNAL AND BACKGROUND SIMULATION}

To simulate the $0 \nu \beta \beta$ process in LHC collisions using mainstream MC tools, we exploit the above observation that the intermediate $\left(\nu_{\ell} \nu_{\ell^{\prime}}^{c}\right)$ current in Fig. 1 can be modeled as an unphysical Majorana neutrino with mass $m_{\ell \ell^{\prime}}=C_{5}^{\ell \ell^{\prime}} v^{2} / \Lambda$. We implement the Lagrangian of Eq. (4) into the FeynRules software package (version 2.3.36) [38-41] by extending the FeynRules implementation of the SM (version 1.4.7) by a single Majorana neutrino $N$ with mass $m_{N}$ and EW boson couplings governed by Eq. (8). We ensure that conventional factors are kept according to Ref. [41]. To account for all $\ell^{ \pm} \ell^{ \pm \pm}$flavor permutations accessible at LHC energies, we make $m_{N}$ an internally calculated quantity that is set by

$$
m_{N}=\left|C_{5}^{e e}+C_{5}^{e \mu}+C_{5}^{e \tau}+C_{5}^{\mu \mu}+C_{5}^{\mu \tau}+C_{5}^{\tau \tau}\right| \frac{v^{2}}{\Lambda} .
$$

Using Refs. [42,43], we extract renormalization and $R_{2}$ counterterms up to the first order in the quantum chromodynamic (QCD) coupling $\alpha_{s}$. Feynman rules are collected into a set of public universal FeynRules output (UFO) libraries that we call the SMWEINBERG libraries.

With this UFO proton collisions are simulated at next-toleading order (NLO) in QCD with the event generator MadGraph5_aMC@NLO (version 2.7.1.2) [44-49]. Parton showering (PS) and modeling of nonperturbative phenomena are handled by PYTHIA8 (version 243) [50]. Hadronlevel events are passed through DELPHES (version 3.4.2) [51] for the simulation of an ATLAS-like detector. Hadron clustering is handled according to the anti- $k_{T}$ algorithm at $R=0.4[52-54]$ as implemented in FastJet $[55,56]$. We tune our simulation tool chain as in the study on $W^{ \pm} W^{ \pm}$ scattering by Ref. [23], whose methodology we also follow to model SM backgrounds.

\section{THE $d=5,0 \nu \beta \beta$ PROCESS AT THE LHC}

In LHC collisions, the $\mathrm{LN}$-violating $0 \nu \beta \beta$ process occurs through the scattering of two same-sign $W$ bosons that are sourced from quarks and antiquarks, and exit as two high- $p_{T}$ jets. At the hadronic level, the collider signature is given by

$$
p p \rightarrow j j \ell^{ \pm} \ell^{\prime \pm}+X,
$$

where $X$ represents the additional hadronic and electromagnetic activity that may exist in the inclusive process.

To identify the dependence of Eq. (10) on the Weinberg operator, we consider the effective $W$ approximation [57-59] and treat the incoming $W^{ \pm} W^{ \pm}$pair as perturbative constituents of the proton. In this limit, we find that the $W^{ \pm} W^{ \pm} \rightarrow \ell^{ \pm} \ell^{ \pm}$subprocess is dominated by the scattering of longitudinal $W$ bosons. After summing over all external helicities, the spin-averaged, parton-level cross section for the $2 \rightarrow 2$ process is given by

$$
\begin{aligned}
& \hat{\sigma}\left(W^{+} W^{+} \rightarrow \ell^{+} \ell^{\prime+}\right) \\
& \quad=\frac{\left(2-\delta_{\ell \ell^{\prime}}\right)}{2 \pi 3^{2}}\left|\frac{C_{5}^{\ell \ell^{\prime}}}{\Lambda}\right|^{2}+\mathcal{O}\left(\frac{m_{W}^{2}}{M_{W W}^{2}}\right) .
\end{aligned}
$$

This shows that like in nuclear experiments the $0 \nu \beta \beta$ rate at the LHC scales as $\sigma \sim\left|m_{\ell \ell^{\prime}}\right|^{2} \propto\left|C_{5}^{\ell \ell^{\prime}} / \Lambda\right|^{2}$.

Using this scaling behavior, we have checked that setting $\Lambda \ll 200 \mathrm{TeV}$ in simulations with the SMWEINBERG UFO will generate unphysical cross sections. This is due to a breakdown of the expansion in Eq. (7), which requires $v^{2} / \Lambda$ to be small compared to the virtuality of the internal $\left(\nu_{\ell} \nu_{\ell^{\prime}}^{c}\right)$ current. For the LHC and beyond, physical rates can be obtained by choosing, for example, $\Lambda=200 \mathrm{TeV}$ and using the relationship 


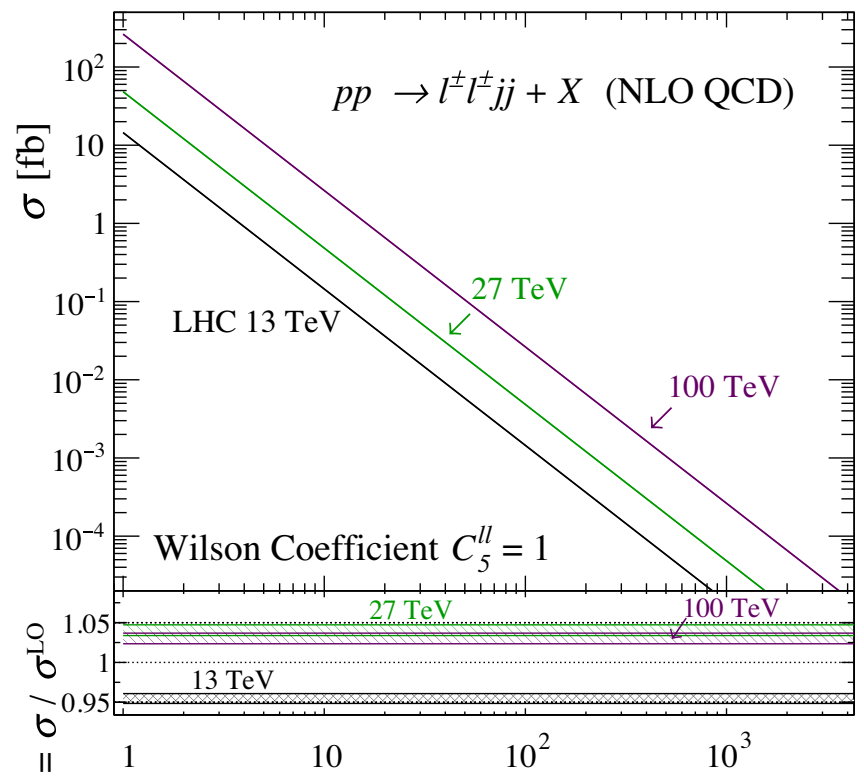

$\Downarrow \quad$ Effective Field Theory Scale, $\Lambda[\mathrm{TeV}]$

FIG. 2. Total cross sections at NLO in QCD (top) and the corresponding NLO $K$-factors (bottom) for the process in Eq. (10), as a function of EFT scale $\Lambda$ with $C_{5}^{\ell \ell^{\prime}}=\delta_{\ell \mu} \delta_{\ell^{\prime} \mu}$, and $\sqrt{s}=13,27,100 \mathrm{TeV}$. Bands represent scale uncertainties.

$$
\sigma(\Lambda)=\sigma(\Lambda=200 \mathrm{TeV}) \times\left(\frac{200 \mathrm{TeV}}{\Lambda}\right)^{2}
$$

Given this guidance, we show in the top of Fig. 2 the hadronic cross section $\sigma$ at NLO in QCD for the full $2 \rightarrow 4$, $0 \nu \beta \beta$ process for $\sqrt{s}=13,27$, and $100 \mathrm{TeV}$, as a function of $\Lambda$, assuming $C_{5}^{\ell \ell^{\prime}}=\delta_{\ell \mu} \delta_{\ell^{\prime} \mu}$. The band thickness for each curve, which reaches $\mathcal{O}(0.5 \%-1.5 \%)$, denotes the nine-point scale uncertainty. While not shown, parton distribution function (PDF) uncertainties reach about $\mathcal{O}(1 \%)$. At $\sqrt{s}=$ $13 \mathrm{TeV}$ and for $\Lambda=10 \mathrm{TeV}$ (or $m_{\mu \mu} \sim 6 \mathrm{GeV}$ ), we find $\sigma \sim 0.14 \mathrm{fb}$. Conversely, at $\sqrt{s}=13(27)[100] \mathrm{TeV}$, we find the rate reaches the $\sigma \sim 1 \mathrm{ab}$ threshold at $\Lambda \sim 120(220)[510] \mathrm{TeV}$, which corresponds to $m_{\mu \mu} \sim$ $500(275)[120] \mathrm{MeV}$. As a measure of the QCD corrections to the cross section, we show in the bottom of Fig. 2 the QCD $K$-factor, defined as the ratio of the NLO and leading order (LO) cross sections. We report that $\mathcal{O}\left(\alpha_{s}\right)$ corrections are mild, reaching $K \sim 0.95-1.05$ across $\sqrt{s}$.

To estimate the LHC's discovery potential of the Weinberg operator, we focus on the $\ell \ell^{\prime}=\mu \mu$ channel with benchmark inputs $C_{5}^{\ell \ell^{\prime}}=\delta_{\ell \mu} \delta_{\ell^{\prime} \mu}$ and $\Lambda=200 \mathrm{TeV}$ (or $m_{\mu \mu} \approx 300 \mathrm{MeV}$ ) and design an analysis inspired by the Run 2 performance of the ATLAS detector $[60,61]$. We employ particle identification requirements on electrons, muons, and jets that are summarized at the top of Table I. For simplicity, we ignore particles originating from pileup interactions as they would mostly be subtracted with dedicated algorithms in real experiments.

To define our signal-enriched region, we demand events to have at least two jets, with the leading pair carrying a large invariant mass, and exactly two muons with the same charge. Events with additional leptons, including hadronically decaying $\tau$ leptons, are vetoed. To further reduce backgrounds, we take into account two qualitative differences between our signal and background processes: (i) unlike SM processes with the same final state, our signal does not contain outgoing neutrinos. As neutrinos go undetected in LHC experiments, their presence gives rise to missing transverse momentum $E_{\mathrm{T}}^{\text {miss }}$, which is defined as the $p_{T}$ recoil against all visible objects. We therefore require that events have a small $E_{\mathrm{T}}^{\text {miss }}$, in accordance with the detector resolution. (ii) Due to the lack of QCD color flowing between the two hadrons in Fig. 1, the hadronic activity is much milder than the QCD and $W^{ \pm} V$ backgrounds. Following past studies [62-64], we impose an upper limit on the ratio $\left(H_{T} / p_{T}^{\mu_{1}}\right)$, where $H_{T}$ is the scalar sum of jet $p_{T}$. To guide our precise cut choice, we plot in Fig. 3 the $\left(H_{T} / p_{T}^{\mu_{1}}\right)$ distribution for our signal and leading backgrounds after applying all other selection cuts.

At this stage, the leading backgrounds consist of mixed EW-QCD production of $W^{ \pm} W^{ \pm} j j$, pure EW production of $W^{ \pm} W^{ \pm} j j$, and the inclusive diboson + jets spectrum $W^{ \pm} V+n j$, with $V \in\left\{\gamma^{*} / Z / Z^{*}\right\}$. We checked that other processes, e.g., $t \bar{t} W^{ \pm}$, do not appreciably survive our event selection. We neglect processes that are especially difficult

TABLE I. Particle identification and signal region definitions.

Particle identification cuts

$p_{T}^{e(\mu)[j]}>10(10)[25] \mathrm{GeV}$, Anti- $k_{T}(R=0.4)$

$\left|\eta^{e(\mu)[j]}\right|<2.5(2.7)[4.5]$

Signal region cuts

$p_{T}^{\mu_{1}\left(\mu_{2}\right)}>27(10) \mathrm{GeV}, n_{\mu}=2, n_{j} \geq 2, n_{e}=n_{\tau^{\mathrm{had}}}=0, Q_{\mu_{1}} \times Q_{\mu_{2}}=1, M\left(j_{1}, j_{2}\right)>700 \mathrm{GeV}$

$E_{\mathrm{T}}^{\text {miss }}<30 \mathrm{GeV},\left(H_{\mathrm{T}} / p_{\mathrm{T}}^{\mu_{1}}\right)<1.6$

Changes to identification and signal cuts at $\sqrt{s}=100 \mathrm{TeV}$

$\left|\eta^{e(\mu)[j]}\right|<4.0(4.0)[5.5], M\left(j_{1}, j_{2}\right)>1 \mathrm{TeV}$

$E_{\mathrm{T}}^{\mathrm{miss}}<20 \mathrm{GeV},\left(H_{\mathrm{T}} / p_{\mathrm{T}}^{\mu_{1}}\right)<0.6$ 


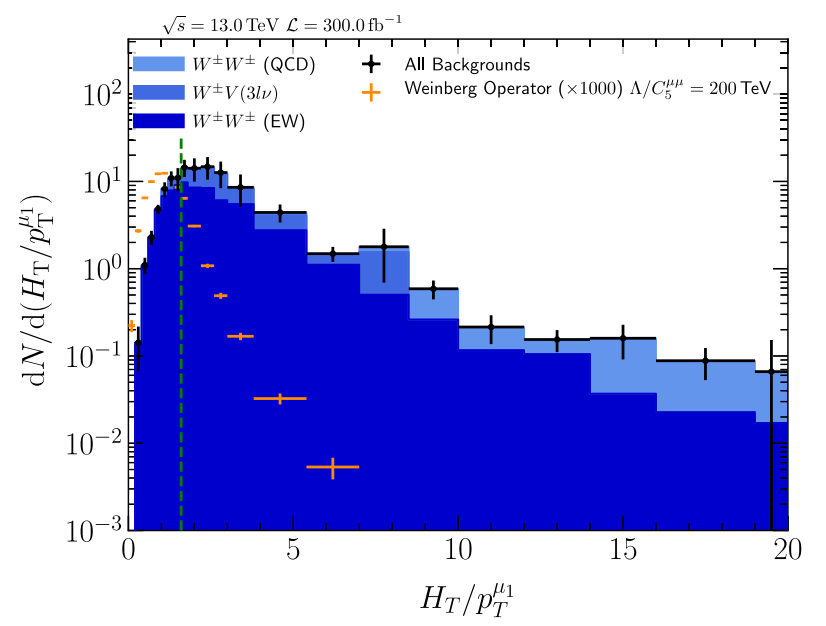

FIG. 3. At $\sqrt{s}=13 \mathrm{TeV}$ with $\mathcal{L}=300 \mathrm{fb}^{-1}$, the $\left(H_{T} / p_{T}^{\mu_{1}}\right)$ distribution at NLO + PS for signal and backgrounds in the signal region, for $C_{5}^{\ell \ell^{\prime}}=\delta_{\ell \mu} \delta_{\ell^{\prime} \mu}$ and $\Lambda=200 \mathrm{TeV}$.

to simulate from MC methods alone. This includes when muons are assigned the wrong charge during event reconstruction. While subdominant for dimuon final states, such backgrounds are relevant for the electron and tau channels [63,65-67]. We account for such backgrounds with a more conservative uncertainty in our background estimate. We summarize our signal region definition in Table I. About $\varepsilon \sim 12 \%$ of generated signal events with $\Lambda=200 \mathrm{TeV}$ pass all identification and signal region cuts. For our inputs, the signal (total background) rate reaches $\sigma \sim 42 \mathrm{zb}(\sigma \sim 25 \mathrm{ab})$.

\section{SENSITIVITY TO THE WEINBERG OPERATOR}

To quantify any excess of events, we apply a Poissoncounting likelihood with a background rate uncertainty that is constrained by an auxiliary Poisson measurement $[68,69]$. Assuming a $\delta_{b}=20 \%$ systematic uncertainty in the background, we estimate the sensitivity at 95\% C.L. to $\left|C_{5}^{\mu \mu}\right| / \Lambda \propto m_{\mu \mu}$ by fixing our signal significance to $Z \approx 2$ and the number of signal events $n_{s}$ to

$$
n_{s}=n_{s}^{0} \times\left|C_{5}^{\mu \mu}\right|^{2}\left(\frac{200 \mathrm{TeV}}{\Lambda}\right)^{2}
$$

where $n_{s}^{0}$ is the number of signal events for our benchmark inputs. We then solve this equality for $\left|C_{5}^{\mu \mu}\right|$. With $\mathcal{L}=300 \mathrm{fb}^{-1}\left(3 \mathrm{ab}^{-1}\right)$, we report that the LHC (HL-LHC) is sensitive at $95 \%$ C.L. to scales below

$$
\Lambda /\left|C_{5}^{\mu \mu}\right| \lesssim 8.3(11) \mathrm{TeV}
$$

These translate into effective $\mu \mu$ Majorana masses of

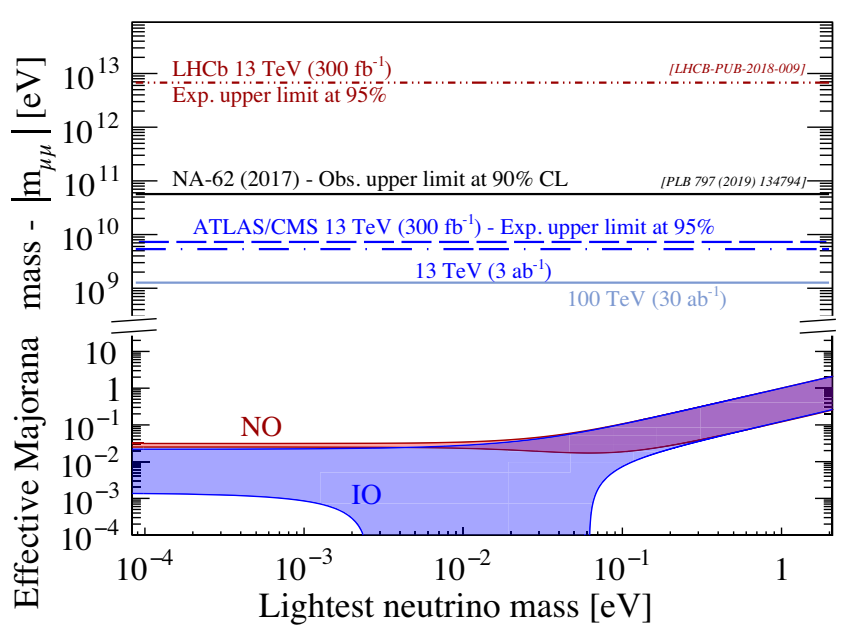

FIG. 4. Projected sensitivity to $\left|m_{\mu \mu}\right|$ at the $\sqrt{s}=13 \mathrm{TeV}$ LHC and a successor proton collider at $\sqrt{s}=100 \mathrm{TeV}$, observed limits set by NA-62 with its 2017 data set [72], and allowed values by best fits to neutrino oscillation data [73].

$$
\left|m_{\mu \mu}\right| \gtrsim 7.3(5.4) \mathrm{GeV} .
$$

With an outlook to potential successors of the HL-LHC [1,2], we estimate the sensitivity of a $\sqrt{s}=100 \mathrm{TeV}$ proton collider. We employ our LHC analysis but with changes listed at the bottom of Table I. We set $\delta_{b}=5 \%$ to account for improved detector resolution and control region modeling. For $\mathcal{L}=30 \mathrm{ab}^{-1}$ of data, we find sensitivity to $\Lambda /\left|C_{5}^{\mu \mu}\right| \lesssim 48 \mathrm{TeV}$ at $95 \%$ C.L. Our precise choice of cuts is for illustration and optimization should be investigated. This is especially relevant as we neglect an $\mathcal{O}(30 \%)$ statistical uncertainty on our $W V^{ \pm}$simulation despite starting from $10^{7}$ NLO + PS events.

As described above, treating the Weinberg operator as an unphysical Majorana fermion is applicable to LN-violating decays of mesons, so long as the expansion in Eq. (7) is satisfied. Using Refs. [70-72], we update the limits and projections on $\left|m_{\mu \mu}\right|$ from $B^{ \pm} \rightarrow \pi^{ \pm} \mu^{\mp} \mu^{\mp}$ and $K^{ \pm} \rightarrow$ $\pi^{\mp} \mu^{ \pm} \mu^{ \pm}$decays. We find that LHCb with $\mathcal{L}=300 \mathrm{fb}^{-1}$ can only probe $\Lambda /\left|C_{5}^{\mu \mu}\right| \lesssim 9 \mathrm{MeV}$ while NA-62 has excluded with its 2017 data set $\Lambda /\left|C_{5}^{\mu \mu}\right| \lesssim 1.1 \mathrm{TeV}$.

Assuming that neutrino masses are described completely at $d=5$, we summarize in Fig. 4 our sensitivities to $\left|m_{\mu \mu}\right|$ in comparison to the values [73] allowed by Eq. (1) (generalized for arbitrary $\ell \ell^{\prime}$ [74]) for normal (NO) and inverse ordering (IO) of neutrino masses. The reach of $W^{ \pm} W^{ \pm}$scattering greatly exceeds our LHCb and NA-62 benchmarks. Nevertheless, improvements at these and similar experiments are anticipated.

\section{CONCLUSIONS}

If the Weinberg operator is present in nature and is accessible at collider energies, then a key prediction are processes that violate $\mathrm{LN}$, such as the $0 \nu \beta \beta$ transition, and 
possibly charged lepton flavor. Motivated by the flavor limitations of nuclear decay experiments, we have investigated the LHC's sensitivity to the Weinberg operator using the $W^{ \pm} W^{ \pm} \rightarrow \ell_{i}^{ \pm} \ell_{j}^{ \pm}$process, which permits muonand tau-flavored final states. We find sensitivity that exceeds representative searches at $B$ - and $K$-meson factories and establishes a complementarity across accelerator facilities in the search for the Weinberg operator.

\section{ACKNOWLEDGMENTS}

J. N. and K. P. acknowledge support by the Deutsche Forschungsgemeinschaft (DFG, German Research Foundation) under Germany's Excellence Strategy-EXC 2121 "Quantum Universe"-390833306. R. R. acknowledges the support of Narodowe Centrum Nauki under Grant No. 2019/34/E/ST2/00186 and the UCLouvain fund "MOVE-IN Louvain." This work received funding from the European Union's Horizon 2020 research and innovation programme as part of the Marie Skłodowska-Curie Innovative Training Network MCnetITN3 (Grant No. 722104), FNRS "Excellence of Science" EOS be.h Project No. 30820817. Computational resources were provided by the supercomputing facilities of the Université catholique de Louvain (CISM/UCL) and the Consortium des Équipements de Calcul Intensif en Fédération Wallonie Bruxelles (CÉCI) funded by the Fond de la Recherche Scientifique de Belgique (F. R. S.-FNRS) under convention 2.5020 .11 and by the Walloon Region.

\section{APPENDIX: TECHNICAL DETAILS ON METHODOLOGY}

In this appendix, we provide additional discussions and details of our methodology.

In a generic gauge, the Higgs field in terms of the EW Goldstone bosons $G^{ \pm, 0}$ is $\sqrt{2} \Phi=\left(-i \sqrt{2} G^{+}\right.$, $\left.v+h+i G^{0}\right)^{T}$. Explicit contraction of $\mathrm{SU}(2)_{L}$ indices then gives

$$
\begin{array}{r}
L_{\ell} \cdot \Phi=L_{\ell}^{i} \varepsilon_{i j} \Phi^{j}=\frac{1}{\sqrt{2}} \nu_{\ell}\left(v+h+i G^{0}\right)+i \ell G^{+}, \\
\Phi \cdot \bar{L}_{\ell}^{c}=-i G^{+} \overline{\ell^{c}}-\frac{1}{\sqrt{2}}\left(v+h+i G^{0}\right) \overline{\nu_{\ell}^{c}} .
\end{array}
$$

This allows us to express the full Weinberg operator as

$$
\begin{aligned}
\mathcal{L}_{5}= & -\frac{C_{5}^{\ell \ell^{\prime}}}{2 \Lambda}\left(v^{2}+2 v h+h h\right) \overline{\nu_{\ell}^{c}} \nu_{\ell^{\prime}} \\
& -\frac{i C_{5}^{\ell \ell^{\prime}}}{\sqrt{2} \Lambda} G^{+}(v+h)\left(\overline{\nu_{\ell}^{c}} \ell^{\prime}+\overline{\ell^{c}} \nu_{\ell^{\prime}}\right) \\
& -\frac{i C_{5}^{\ell \ell^{\prime}}}{\Lambda} G^{0}(v+h) \overline{\nu_{\ell}^{c}} \nu_{\ell^{\prime}}
\end{aligned}
$$

$$
\begin{aligned}
& +\frac{C_{5}^{\ell \ell^{\prime}}}{2 \Lambda}\left(2 G^{+} G^{+} \overline{\ell^{c}} \ell^{\prime}+G^{0} G^{0} \overline{\nu_{\ell}^{c}} \nu_{\ell^{\prime}}\right) \\
& +\frac{C_{5}^{\ell \ell^{\prime}}}{\sqrt{2} \Lambda} G^{0} G^{+}\left(\overline{\nu_{\ell}^{c}} \ell^{\prime}+\overline{\ell^{c}} \nu_{\ell^{\prime}}\right)+\text { H.c. }
\end{aligned}
$$

Here and below, the Hermitian conjugate is understood to apply to the full expression, not simply the final line.

With this, the interaction Lagrangian by which we extend the SM Lagrangian in the SMWEINBERG UFO is

$$
\begin{gathered}
\Delta \mathcal{L}=-\frac{g_{W}}{\sqrt{2}} W_{\mu}^{+} \sum_{\ell=e}^{\tau} \bar{N} \gamma^{\mu} P_{L} \ell^{-} \\
-\frac{g_{W}}{2 \cos \theta_{W}} Z_{\mu} \sum_{\ell=e}^{\tau} \bar{N} \gamma^{\mu} P_{L} \nu_{\ell} \\
-\frac{g_{W} m_{N}}{2 m_{W}} h\left(1+\frac{g_{W}}{4 m_{W}} h\right) \sum_{\ell=e}^{\tau} \bar{N} P_{L} \nu_{\ell} \\
-i \frac{g_{W} m_{N}}{2 \sqrt{2} m_{W}} G^{+}\left(1+\frac{g_{W}}{2 m_{W}} h\right) \sum_{\ell=e}^{\tau}\left(\bar{N} P_{L} \ell+\overline{\ell^{c}} P_{L} N\right) \\
-i \frac{g_{W} m_{N}}{2 m_{W}} G^{0}\left(1+\frac{g_{W}}{2 m_{W}} h\right) \sum_{\ell=e}^{\tau} \bar{N} P_{L} \nu_{\ell} \\
+\frac{g_{W}^{2} m_{N}}{8 m_{W}^{2}}\left(2 G^{+} G^{+} \sum_{\ell,}^{\tau} \overline{\ell^{\prime}=e}\right. \\
\left.+\frac{g_{W}^{2} m_{N}}{4 \sqrt{2} m_{W}^{2}} G_{L}^{0} \ell^{\prime}+G^{0} G^{0} \sum_{\ell=e}^{\tau} \bar{N} P_{L} \nu_{\ell}\right)
\end{gathered}
$$

To further understand the identification in Eq. (7), we recall that the fermions in the $\mathrm{LN}$-violating $\left(\ell^{+} \nu_{\ell} \nu_{\ell^{\prime}}^{c} \ell^{\prime+}\right)$ current in Fig. 1 experience an additional parity inversion beyond the standard $\mathrm{SU}(2)_{L}$ chiral couplings [31,32]. In terms of Feynman rules $[33,34]$, this manifests as a chiral inversion of the $\left(W \ell^{\prime} \nu_{\ell^{\prime}}^{c}\right)$ vertex, i.e., $\gamma^{\beta} P_{L} \rightarrow \gamma^{\beta} P_{R}$. In the absence of additional new physics, this ensures $[35,36]$ the presence of the $P_{R / L}$ projection operators that envelope the $\left(\nu_{\ell} \nu_{\ell^{\prime}}^{c}\right)$ current in Eq. (6), and hence that the $\left(\nu_{\ell} \nu_{\ell^{\prime}}^{c}\right)$ current propagates in the RH helicity state.

The model's input parameters along with their FeynRules and Les Houches [75] information are summarized in Table II. The syntax used to import the UFO into MadGraph5_aMC@NLO and simulate the process 
TABLE II. Parameters for the SMWEINBERG UFO.

\begin{tabular}{lcccc}
\hline \hline Parameter & FR name & Type & LH block & LH counter \\
\hline$\Lambda$ & Lambda & External (real) & NUPHYSICS & 1 \\
$C_{5}^{\ell \ell^{\prime}}$ & $\mathrm{Cll}$ & External (real) & NUPHYSICS & $2-7$ \\
$m_{N}$ & $\mathrm{mN}$ & Internal (real) & MASS & 9900012 \\
$\Gamma_{N}$ & $\mathrm{wN}$ & Internal (real) & WIDTH & 9900012 \\
\hline \hline
\end{tabular}

$$
q_{1} q_{2} \rightarrow q_{1}^{\prime} q_{2}^{\prime} \mu^{ \pm} \mu^{ \pm}
$$

where $q$ is any light quark or antiquark, at NLO is

import model sMWeinbergNLO

generate $\mathrm{p} \mathrm{p}>\mathrm{mu}+\mathrm{mu}+\mathrm{j} \mathrm{QED}=4 \mathrm{QCD}=0$

$\$ \$ \mathrm{~W}+\mathrm{w}-[\mathrm{QCD}]$

add process p p > mu-mu- j j $\mathrm{QED}=4 \mathrm{QCD}=0$

$\$ \$ \mathrm{~W}+\mathrm{w}-[\mathrm{QCD}]$

For SM inputs, we approximate the quark sector by $n_{f}=$ 5 massless quarks that do not mix. Values of couplings and masses are set to global averages reported in the 2020 Particle Data Group review [76],

$$
\begin{aligned}
m_{t}\left(m_{t}\right) & =172.76 \mathrm{GeV}, \quad m_{h}=125.1 \mathrm{GeV}, \\
M_{Z} & =91.1876 \mathrm{GeV}, \quad \alpha_{\mathrm{QED}}^{-1}\left(M_{Z}\right)=127.952, \\
G_{F} & =1.1663787 \times 10^{-5} \mathrm{GeV}^{-2}, \quad \alpha_{s}\left(M_{Z}\right)=0.118 .
\end{aligned}
$$

We employ the NNPDF3.1 NLO + LUXqed parton distribution function set (lhaid=324900) [77-79], with scale evolution driven by LHAPDF [80], and PDF uncertainties are extracted using the replica method $[79,80]$. We fix the collinear factorization $\left(\mu_{f}\right)$, QCD renormalization $\left(\mu_{r}\right)$, and shower matching $\left(\mu_{s}\right)$ scales to the default values in Ref. [45]. The uncertainty in choosing $\mu_{f}$ and $\mu_{r}$ is quantified by scaling their baseline values by factors of $0.5,1$, and 2 to obtain a nine-point uncertainty band.

As a check of the SMWEINBERG UFO, we consider the amplitudes for the $W^{ \pm}\left(p_{1}^{W}, \lambda_{1}^{W}\right) W^{ \pm}\left(p_{2}^{W}, \lambda_{2}^{W}\right) \rightarrow \ell^{ \pm}\left(p_{1}^{\ell}\right.$, $\left.\lambda_{1}^{\ell}\right) \ell^{\prime \pm}\left(p_{2}^{\ell}, \lambda_{1}^{\ell}\right)$ process. Explicit calculation reveals that in the high-energy limit, i.e., when $M_{W W}^{2}=\left(p_{1}^{W}+p_{2}^{W}\right)^{2} \gg$ $m_{W}^{2}$, the $2 \rightarrow 2$ process is dominated by the scattering of two longitudinally polarized $W^{ \pm}$bosons. For the $\left(\lambda_{1}^{W}, \lambda_{2}^{W}\right)=$ $(0,0)$ helicity configuration, the exact helicity amplitude is

$$
\begin{gathered}
-i \mathcal{M}\left(W_{0}^{+} W_{0}^{+} \rightarrow \ell_{R}^{+} \ell_{R}^{\prime+}\right)=-i \mathcal{M}_{t}+-i \mathcal{M}_{u}, \\
-i \mathcal{M}_{t}=i e^{-i \phi_{1}}\left(\frac{C_{5}^{\ell \ell^{\prime}}}{\Lambda}\right)\left(\frac{M_{W W}^{3}}{t}\right) \\
\times\left[1-2 r_{W}-\sqrt{1-4 r_{W}} \cos \theta_{1}\right],
\end{gathered}
$$

$$
\begin{aligned}
-i \mathcal{M}_{u}= & i e^{-i \phi_{1}}\left(\frac{C_{5}^{\ell \ell^{\prime}}}{\Lambda}\right)\left(\frac{M_{W W}^{3}}{t}\right) \\
& \times\left[1-2 r_{W}+\sqrt{1-4 r_{W}} \cos \theta_{1}\right],
\end{aligned}
$$

where $r_{W}=m_{W}^{2} / M_{W W}^{2} ; \theta_{1}$ and $\phi_{1}$ are, respectively, the polar and azimuthal angles of $\ell\left(p_{1}^{\ell}\right)$ in the $(W W)$ frame, and the kinematic invariants are defined by $t=\left(p_{1}^{W}-p_{1}^{\ell}\right)^{2}$ and $u=\left(p_{1}^{W}-p_{2}^{\ell}\right)^{2}$. Further evaluation of $t$ and $u$ results in the somewhat simple expression

$$
\mathcal{M}\left(W_{0}^{+} W_{0}^{+} \rightarrow \ell_{R}^{+} \ell_{R}^{++}\right)=e^{-i \phi_{1}}\left(\frac{4 C_{5}^{\ell \ell^{\prime}} M_{W W}}{\Lambda}\right) .
$$

The $J=0$ partial wave is subsequently given by

$$
\begin{aligned}
a_{J=0} & =\frac{1}{32 \pi} \int_{-1}^{1} d \cos \theta_{1} \mathcal{M}\left(W_{0}^{+} W_{0}^{+} \rightarrow \ell_{R}^{+} \ell_{R}^{\prime+}\right) \\
& =\frac{1}{4 \pi} \frac{C_{5}^{\ell \ell^{\prime}} M_{W W}}{\Lambda} .
\end{aligned}
$$

Since $s$-wave perturbative unitarity requires that $\left|a_{J}\right|<1$, one obtains the constraint that

$$
\left|C_{5}\right| M_{W W}<4 \pi \Lambda \text {. }
$$

After evaluating the exact helicity amplitude for each $\left(\lambda_{1}^{W}, \lambda_{2}^{W}\right)$ permutation, taking their sum, and then taking the high-energy limit, we obtain

$$
\begin{aligned}
& \sum_{\left\{\lambda^{W}, \lambda^{\ell}\right\}}\left|\mathcal{M}\left(W^{+} W^{+} \rightarrow \ell^{+} \ell^{\prime+}\right)\right|^{2} \\
& \quad=8\left(2-\delta_{\ell \ell^{\prime}}\right)\left|\frac{C_{5}^{\ell \ell^{\prime}} M_{W W}}{\Lambda}\right|^{2}+\mathcal{O}\left(\frac{m_{W}^{2}}{M_{W W}^{2}}\right) .
\end{aligned}
$$

The Kronecker $\delta$ accounts for the $1 / 2$ ! symmetry factor needed for amplitudes with identical final-state particles. This implies a totally differential cross section of

$\frac{d \hat{\sigma}}{d \cos \theta_{1} d \phi_{1}}=\frac{\left(2-\delta_{\ell \ell^{\prime}}\right)}{8 \pi^{2} 3^{2}}\left|\frac{C_{5}^{\ell \ell^{\prime}}}{\Lambda}\right|^{2}+\mathcal{O}\left(\frac{m_{W}^{2}}{M_{W W}^{2}}\right)$.

Integration over the full solid angle recovers Eq. (11).

Using Eq. (11) as a check of the SMWEINBERG UFO, we list in Table III the total $2 \rightarrow 4$, hadronic cross section $\sigma$ for Eq. (10) at NLO in QCD for representative cutoff scales $\Lambda$, assuming a Wilson coefficient of $C_{5}^{\ell \ell^{\prime}}=\delta_{\ell_{\mu}} \delta_{\ell^{\prime} \mu}$, for the $\sqrt{s}=13 \mathrm{TeV}$ LHC and proposed experiments at $\sqrt{s}=27$ and $100 \mathrm{TeV}$. Also listed are the corresponding (unphysical) Majorana neutrino mass $m_{N}$, as defined by Eq. (9), the nine-point scale uncertainty $\delta_{\text {Scale }}$, the parton distribution 
TABLE III. The total cross section at NLO in QCD for the process in Eq. (10) for various choices of EFT scale $\Lambda$ and collider energy $\sqrt{s}$ at a Wilson coefficient $C_{5}^{\ell \ell^{\prime}}=\delta_{\ell \mu} \delta_{\ell^{\prime} \mu}$, and the corresponding mass $m_{N}$, uncertainties, and NLO $K$-factor.

\begin{tabular}{|c|c|c|c|c|c|c|}
\hline$\Lambda(\mathrm{TeV})$ & $m_{N}(\mathrm{GeV})$ & $\sqrt{s}(\mathrm{TeV})$ & $\sigma^{\mathrm{NLO}}(\mathrm{ab})$ & $\delta_{\text {Scale }}$ & $\delta_{\mathrm{PDF}}$ & $K^{\mathrm{NLO}}$ \\
\hline 10 & 6 & 13 & 133 & ${ }_{-0.8 \%}^{+0.8 \%}$ & ${ }_{-0.9 \%}^{+0.9 \%}$ & 0.968 \\
\hline 100 & 0.6 & 13 & 1.42 & $\begin{array}{l}+1.0 \% \\
-0.6 \%\end{array}$ & $\begin{array}{l}+0.9 \% \\
-0.9 \%\end{array}$ & 0.978 \\
\hline 200 & 0.3 & 13 & 0.361 & $\begin{array}{l}+0.7 \% \\
-0.6 \%\end{array}$ & $\begin{array}{l}+1.0 \% \\
-1.0 \%\end{array}$ & 0.952 \\
\hline 400 & 0.15 & 13 & 0.0904 & $\begin{array}{l}-0.0 \% \% \\
+0.6 \%\end{array}$ & $\begin{array}{l}-1.0 \% \% \\
+0.9 \%\end{array}$ & 0.988 \\
\hline 200 & 0.3 & 27 & 1.21 & $\begin{array}{l}-0.9 \% \\
+0.9 \%\end{array}$ & $\begin{array}{l}+0.9 \% \\
+0.9 \%\end{array}$ & 1.04 \\
\hline 200 & 0.3 & 100 & 6.56 & $\begin{array}{l}-1.0 \% \\
+1.2 \% \\
-1.2 \% \\
\end{array}$ & $\begin{array}{r}-0.9 \% \\
+0.9 \% \\
-0.9 \% \\
\end{array}$ & 1.03 \\
\hline
\end{tabular}

function uncertainty $\delta_{\mathrm{PDF}}$, and the QCD $K$-factor, which is defined as the ratio $K=\sigma / \sigma^{L O}$, where $\sigma^{\mathrm{LO}}$ is the $\mathrm{LO}$ rate.

For $\Lambda=10 \mathrm{TeV}$ and $100 \mathrm{TeV}$ at $\sqrt{s}=13 \mathrm{TeV}$, we observe a cross section scaling of $\sigma(\Lambda=10 \mathrm{TeV}) /$ $\sigma(\Lambda=100 \mathrm{TeV}) \sim 93$, undershooting the $100 \times$ scaling expected from Eq. (11). We attribute this to a breakdown of Eq. (7), which requires the mass $m_{N} \sim v^{2} / \Lambda$ to be small compared to the virtuality of $\left(\nu_{\ell} \nu_{\ell^{\prime}}^{c}\right)$. At larger $\Lambda$, we find, for example, that $\sigma(\Lambda=100 \mathrm{TeV}) / \sigma(\Lambda=200 \mathrm{TeV}) \sim 3.93$ and $\sigma(\Lambda=200 \mathrm{TeV}) / \sigma(\Lambda=400 \mathrm{TeV}) \sim 3.99$, indicating behavior more inline with Eq. (11). We conclude that choices of $\Lambda \gtrsim 200 \mathrm{TeV}$ generate sufficiently small $m_{N}$ so that Eq. (7) remains valid for $\sqrt{s} \gtrsim 13 \mathrm{TeV}$.

Assuming benchmark signal inputs of $\Lambda=200 \mathrm{TeV}$ and $C_{5}^{\ell \ell^{\prime}}=\delta_{\ell \mu} \delta_{\ell^{\prime} \mu}$, we summarize in Table IV the expected number of signal and background events after all cuts for the LHC (HL-LHC) with $\mathcal{L}=300 \mathrm{fb}^{-1}\left(3 \mathrm{ab}^{-1}\right)$. To quantify the LHC's sensitivity to the Weinberg operator, we define our signal significance $Z$ as $[68,69]$

$$
\begin{gathered}
Z=\frac{\left(n-n_{b}\right)}{\left|n-n_{b}\right|} \sqrt{2\left[n \log x-\frac{n_{b}^{2}}{\delta_{b}^{2}} \log y\right]}, \text { with } \\
x=\frac{n\left(n_{b}+\delta_{b}^{2}\right)}{n_{b}^{2}+n \delta_{b}^{2}}, \quad \text { and } \quad y=1+\frac{\delta_{b}^{2}\left(n-n_{b}\right)}{n_{b}\left(n_{b}+\delta_{b}^{2}\right)} .
\end{gathered}
$$

TABLE IV. For benchmark signal inputs of $\Lambda=200 \mathrm{TeV}$ and $C_{5}^{\ell \ell^{\prime}}=\delta_{\ell \mu} \delta_{\ell^{\prime} \mu}$, the expected number of background and $0 \nu \beta \beta$ signal events in the signal region with $\mathcal{L}=300 \mathrm{fb}^{-1}\left(3 \mathrm{ab}^{-1}\right)$.

\begin{tabular}{llllll}
\hline Collider & QCD $W^{ \pm} W^{ \pm} j j$ & EW $W^{ \pm} W^{ \pm} j j$ & $W^{ \pm} V$ & Total & Signal
\end{tabular}

\begin{tabular}{llcccl}
\hline LHC & $<0.01$ & 6.40 & 1.16 & 7.56 & 0.013 \\
HL-LHC & $<0.01$ & 64.0 & 11.6 & 75.5 & 0.13 \\
\hline
\end{tabular}

Here, $n=n_{s}+n_{b}$ is the total number of observed events, $n_{s}\left(n_{b}\right)$ is the predicted number of signal (background) events, and $\delta_{b}$ is the uncertainty on $n_{b}$.

Under the parametrization of the PMNS matrix

$$
\begin{aligned}
U_{\mathrm{PMNS}}= & \left(\begin{array}{ccc}
1 & 0 & 0 \\
0 & c_{23} & s_{23} \\
0 & -s_{23} & c_{23}
\end{array}\right) \cdot\left(\begin{array}{ccc}
c_{13} & 0 & s_{13} e^{-i \delta_{\mathrm{CP}}} \\
0 & 1 & 0 \\
-s_{13} e^{i \delta_{\mathrm{CP}}} & 0 & c_{13}
\end{array}\right) \\
& \cdot\left(\begin{array}{ccc}
c_{12} & s_{12} & 0 \\
-s_{12} & c_{12} & 0 \\
0 & 0 & 1
\end{array}\right) \cdot\left(\begin{array}{ccc}
e^{i \eta_{1}} & 0 & 0 \\
0 & e^{i \eta_{2}} & 0 \\
0 & 0 & 1
\end{array}\right), \quad(\mathrm{A} 28)
\end{aligned}
$$

where $c_{i j}=\cos \theta_{i j}, s_{i j}=\sin \theta_{i j}$, and $\delta_{\mathrm{CP}}, \eta_{1}$, and $\eta_{2}$ are imaginary phases, the regions for the allowed effective Majorana massses $\left|m_{e e}\right|$ and $\left|m_{\mu \mu}\right|$ are obtained with [74]

$$
\begin{gathered}
U_{e 1}=c_{12} c_{13} e^{i \eta_{1}}, \\
U_{e 2}=s_{12} c_{13} e^{i \eta_{2}}, \\
U_{e 3}=s_{13} e^{-i \delta_{\mathrm{CP}}}, \\
U_{\mu 1}=-s_{12} c_{23} e^{i \eta_{1}}-c_{12} s_{13} s_{23} e^{i\left(\delta_{\mathrm{CP}}+\eta_{1}\right)}, \\
U_{\mu 2}=c_{12} c_{23} e^{i \eta_{2}}-s_{12} s_{13} s_{23} e^{i\left(\delta_{\mathrm{CP}}+\eta_{2}\right)}, \\
U_{\mu 3}=c_{13} s_{23} .
\end{gathered}
$$

[1] R. K. Ellis et al., Physics briefing book: Input for the European strategy for particle physics update 2020, arXiv:1910.11775.

[2] European Strategy Group, 2020 Update of the European Strategy for Particle Physics (CERN Council, Geneva, 2020), https://doi.org/10.17181/ESU2020.

[3] A. Atre, T. Han, S. Pascoli, and B. Zhang, The search for heavy Majorana neutrinos, J. High Energy Phys. 05 (2009) 030.

[4] W. Rodejohann, Neutrino-less double beta decay and particle physics, Int. J. Mod. Phys. E 20, 1833 (2011).
[5] S. M. Bilenky and C. Giunti, Neutrinoless double-beta decay: A probe of physics beyond the standard model, Int. J. Mod. Phys. A 30, 1530001 (2015).

[6] F. F. Deppisch, P. B. Dev, and A. Pilaftsis, Neutrinos and collider physics, New J. Phys. 17, 075019 (2015).

[7] Y. Cai, J. Herrero-García, M. A. Schmidt, A. Vicente, and R.R. Volkas, From the trees to the forest: A review of radiative neutrino mass models, Front. Phys. 5, 63 (2017). 
[8] Y. Cai, T. Han, T. Li, and R. Ruiz, Lepton number violation: Seesaw models and their collider tests, Front. Phys. 6, 40 (2018).

[9] V. Cirigliano, W. Dekens, J. de Vries, M. Graesser, and E. Mereghetti, A neutrinoless double beta decay master formula from effective field theory, J. High Energy Phys. 12 (2018) 097.

[10] M. J. Dolinski, A. W. Poon, and W. Rodejohann, Neutrinoless double-beta decay: Status and prospects, Annu. Rev. Nucl. Part. Sci. 69, 219 (2019).

[11] M. Agostini et al. (GERDA Collaboration), Final Results of GERDA on the Search for Neutrinoless Double- $\beta$ Decay, Phys. Rev. Lett. 125, 252502 (2020).

[12] H. Klapdor-Kleingrothaus, H. Pas, and A. Smirnov, Neutrino mass spectrum and neutrinoless double beta decay, Phys. Rev. D 63, 073005 (2001).

[13] E. Ma, Pathways to Naturally Small Neutrino Masses, Phys. Rev. Lett. 81, 1171 (1998).

[14] F. Bonnet, M. Hirsch, T. Ota, and W. Winter, Systematic study of the $d=5$ Weinberg operator at one-loop order, J. High Energy Phys. 07 (2012) 153.

[15] S. Weinberg, Baryon and Lepton Nonconserving Processes, Phys. Rev. Lett. 43, 1566 (1979).

[16] J. Jenkins, Minimally allowed beta beta $0 \mathrm{nu}$ rates from approximate flavor symmetries, Phys. Rev. D 79, 113004 (2009).

[17] T. Asaka, H. Ishida, and K. Tanaka, Hiding neutrinoless double beta decay in the minimal seesaw mechanism, Phys. Rev. D 103, 015014 (2021).

[18] T. Asaka, H. Ishida, and K. Tanaka, What if a specific neutrinoless double beta decay is absent, arXiv:2012.13186.

[19] T. Asaka, H. Ishida, and K. Tanaka, Neutrinoless double beta decays tell nature of right-handed neutrinos, arXiv: 2101.12498.

[20] D. A. Dicus, D. D. Karatas, and P. Roy, Lepton nonconservation at supercollider energies, Phys. Rev. D 44, 2033 (1991).

[21] A. Datta, M. Guchait, and A. Pilaftsis, Probing lepton number violation via majorana neutrinos at hadron supercolliders, Phys. Rev. D 50, 3195 (1994).

[22] M. Aoki, K. Enomoto, and S. Kanemura, Probing charged lepton number violation via $\ell^{ \pm} \ell^{\prime \pm} W^{\mp} W^{\mp}$, Phys. Rev. D 101, 115019 (2020).

[23] B. Fuks, J. Neundorf, K. Peters, R. Ruiz, and M. Saimpert, Majorana neutrinos in same-sign $W^{ \pm} W^{ \pm}$scattering at the LHC: Breaking the TeV barrier, Phys. Rev. D 103, 055005 (2021).

[24] T. G. Rizzo, Inverse neutrinoless double beta decay, Phys. Lett. 116B, 23 (1982).

[25] G. Belanger, F. Boudjema, D. London, and H. Nadeau, Inverse neutrinoless double beta decay revisited, Phys. Rev. D 53, 6292 (1996).

[26] https://feynrules.irmp.ucl.ac.be/wiki/SMWeinberg

[27] S. Weinberg, Effective gauge theories, Phys. Lett. 91B, 51 (1980).

[28] B. Grzadkowski, M. Iskrzynski, M. Misiak, and J. Rosiek, Dimension-six terms in the standard model Lagrangian, J. High Energy Phys. 10 (2010) 085.
[29] A. Kobach, Baryon number, lepton number, and operator dimension in the standard model, Phys. Lett. B 758, 455 (2016).

[30] D. Wyler and L. Wolfenstein, Massless neutrinos in leftright symmetric models, Nucl. Phys. B218, 205 (1983).

[31] B. Kayser, Majorana neutrinos and their electromagnetic properties, Phys. Rev. D 26, 1662 (1982).

[32] R. N. Mohapatra and P. B. Pal, Massive neutrinos in physics and astrophysics, World Sci. Lect. Notes Phys. 60, 397 (1998); 72, 451 (2004).

[33] A. Denner, H. Eck, O. Hahn, and J. Kublbeck, Feynman rules for fermion number violating interactions, Nucl. Phys. B387, 467 (1992).

[34] A. Denner, H. Eck, O. Hahn, and J. Kublbeck, Compact Feynman rules for Majorana fermions, Phys. Lett. B 291, 278 (1992).

[35] T. Han, I. Lewis, R. Ruiz, and Z.-G. Si, Lepton number violation and $W^{\prime}$ chiral couplings at the LHC, Phys. Rev. D 87, 035011 (2013).

[36] R. Ruiz, Quantitative study on helicity inversion in Majorana neutrino decays at the LHC, Phys. Rev. D 103, 015022 (2021).

[37] F. del Aguila and J. Aguilar-Saavedra, Distinguishing seesaw models at LHC with multi-lepton signals, Nucl. Phys. B813, 22 (2009).

[38] N. D. Christensen and C. Duhr, FeynRules-Feynman rules made easy, Comput. Phys. Commun. 180, 1614 (2009).

[39] N. D. Christensen, P. de Aquino, C. Degrande, C. Duhr, B. Fuks, M. Herquet, F. Maltoni, and S. Schumann, A comprehensive approach to new physics simulations, Eur. Phys. J. C 71, 1541 (2011).

[40] C. Degrande, C. Duhr, B. Fuks, D. Grellscheid, O. Mattelaer, and T. Reiter, UFO-The universal FeynRules output, Comput. Phys. Commun. 183, 1201 (2012).

[41] A. Alloul, N. D. Christensen, C. Degrande, C. Duhr, and B. Fuks, FeynRules 2.0-A complete toolbox for tree-level phenomenology, Comput. Phys. Commun. 185, 2250 (2014).

[42] C. Degrande, Automatic evaluation of UV and R2 terms for beyond the Standard Model Lagrangians: A proof-ofprinciple, Comput. Phys. Commun. 197, 239 (2015).

[43] T. Hahn, Generating Feynman diagrams and amplitudes with FeynArts 3, Comput. Phys. Commun. 140, 418 (2001).

[44] T. Stelzer and W. Long, Automatic generation of tree level helicity amplitudes, Comput. Phys. Commun. 81, 357 (1994).

[45] J. Alwall, R. Frederix, S. Frixione, V. Hirschi, F. Maltoni, O. Mattelaer, H. S. Shao, T. Stelzer, P. Torrielli, and M. Zaro, The automated computation of tree-level and next-toleading order differential cross sections, and their matching to parton shower simulations, J. High Energy Phys. 07 (2014) 079.

[46] S. Frixione and B. R. Webber, Matching NLO QCD computations and parton shower simulations, J. High Energy Phys. 06 (2002) 029.

[47] R. Frederix, S. Frixione, F. Maltoni, and T. Stelzer, Automation of next-to-leading order computations in QCD: The FKS subtraction, J. High Energy Phys. 10 (2009) 003. 
[48] V. Hirschi, R. Frederix, S. Frixione, M. V. Garzelli, F. Maltoni, and R. Pittau, Automation of one-loop QCD corrections, J. High Energy Phys. 05 (2011) 044.

[49] V. Hirschi and O. Mattelaer, Automated event generation for loop-induced processes, J. High Energy Phys. 10 (2015) 146.

[50] T. Sjöstrand, S. Ask, J. R. Christiansen, R. Corke, N. Desai, P. Ilten, S. Mrenna, S. Prestel, C. O. Rasmussen, and P.Z. Skands, An introduction to PYTHIA 8.2, Comput. Phys. Commun. 191, 159 (2015).

[51] J. de Favereau, C. Delaere, P. Demin, A. Giammanco, V. Lemaître, A. Mertens, and M. Selvaggi (DELPHES 3 Collaboration), DELPHES 3, A modular framework for fast simulation of a generic collider experiment, J. High Energy Phys. 02 (2014) 057.

[52] S. Catani, Y. L. Dokshitzer, M. Seymour, and B. Webber, Longitudinally invariant $K_{t}$ clustering algorithms for hadron hadron collisions, Nucl. Phys. B406, 187 (1993).

[53] S. D. Ellis and D. E. Soper, Successive combination jet algorithm for hadron collisions, Phys. Rev. D 48, 3160 (1993).

[54] M. Cacciari, G. P. Salam, and G. Soyez, The anti- $k_{t}$ jet clustering algorithm, J. High Energy Phys. 04 (2008) 063.

[55] M. Cacciari and G. P. Salam, Dispelling the $N^{3}$ myth for the $k_{t}$ jet-finder, Phys. Lett. B 641, 57 (2006).

[56] M. Cacciari, G. P. Salam, and G. Soyez, FastJet user manual, Eur. Phys. J. C 72, 1896 (2012).

[57] S. Dawson, The effective W approximation, Nucl. Phys. B249, 42 (1985).

[58] G. L. Kane, W. Repko, and W. Rolnick, The effective W+-, Z0 approximation for high-energy collisions, Phys. Lett. B 148, 367 (1984).

[59] Z. Kunszt and D. E. Soper, On the validity of the effective $W$ approximation, Nucl. Phys. B296, 253 (1988).

[60] ATLAS Collaboration, The ATLAS experiment at the CERN large hadron collider, J. Instrum. 3, S08003 (2008) .

[61] ATLAS Collaboration, ATLAS insertable B-layer technical design report, Report No. ATLAS-TDR-19, 2010.

[62] S. Pascoli, R. Ruiz, and C. Weiland, Safe jet vetoes, Phys. Lett. B 786, 106 (2018).

[63] S. Pascoli, R. Ruiz, and C. Weiland, Heavy neutrinos with dynamic jet vetoes: Multilepton searches at $\sqrt{s}=14,27$, and $100 \mathrm{TeV}$, J. High Energy Phys. 06 (2019) 049.

[64] B. Fuks, K. Nordström, R. Ruiz, and S. L. Williamson, Sleptons without hadrons, Phys. Rev. D 100, 074010 (2019).

[65] G. Aad et al. (ATLAS Collaboration), Search for supersymmetry at $\sqrt{s}=13 \mathrm{TeV}$ in final states with jets and two same-sign leptons or three leptons with the ATLAS detector, Eur. Phys. J. C 76, 259 (2016).
[66] E. Alvarez, D. A. Faroughy, J. F. Kamenik, R. Morales, and A. Szynkman, Four tops for LHC, Nucl. Phys. B915, 19 (2017).

[67] CMS Collaboration, Jet $\rightarrow$ Tau misidentification probability with $2016 \mathrm{pp}$ collision data at $\sqrt{s}=13 \mathrm{TeV}$, Report No. , 2017.

[68] ATLAS Collaboration, Formulae for Estimating Significance, CERN Report No. ATL-PHYS-PUB-2020-025, 2020, https://cds.cern.ch/record/2736148.

[69] R. D. Cousins, J. T. Linnemann, and J. Tucker, Evaluation of three methods for calculating statistical significance when incorporating a systematic uncertainty into a test of the background-only hypothesis for a Poisson process, Nucl. Instrum. Methods Phys. Res., Sect. A 595, 480 (2008).

[70] A. Atre, V. Barger, and T. Han, Upper bounds on leptonnumber violating processes, Phys. Rev. D 71, 113014 (2005).

[71] R. Aaij et al. (LHCb Collaboration), Physics case for an LHCb upgrade II-Opportunities in flavour physics, and beyond, in the HL-LHC era, arXiv:1808.08865.

[72] E. Cortina Gil et al. (NA62 Collaboration), Searches for lepton number violating $K^{+}$decays, Phys. Lett. B 797, 134794 (2019).

[73] I. Esteban, M. Gonzalez-Garcia, M. Maltoni, T. Schwetz, and A. Zhou, The fate of hints: Updated global analysis of three-flavor neutrino oscillations, J. High Energy Phys. 09 (2020) 178.

[74] M. Frigerio and A. Y. Smirnov, Structure of neutrino mass matrix and $C P$ violation, Nucl. Phys. B640, 233 (2002).

[75] P. Z. Skands et al., SUSY Les Houches accord: Interfacing SUSY spectrum calculators, decay packages, and event generators, J. High Energy Phys. 07 (2004) 036.

[76] P. Zyla et al. (Particle Data Group), Review of particle physics, Prog. Theor. Exp. Phys. 2020, 083 C01 (2020).

[77] A. Manohar, P. Nason, G. P. Salam, and G. Zanderighi, How Bright is the Proton? A Precise Determination of the Photon Parton Distribution Function, Phys. Rev. Lett. 117, 242002 (2016).

[78] A. V. Manohar, P. Nason, G. P. Salam, and G. Zanderighi, The photon content of the proton, J. High Energy Phys. 12 (2017) 046.

[79] V. Bertone, S. Carrazza, N. P. Hartland, and J. Rojo (NNPDF Collaboration), Illuminating the photon content of the proton within a global PDF analysis, SciPost Phys. 5, 008 (2018).

[80] A. Buckley, J. Ferrando, S. Lloyd, K. Nordström, B. Page, M. Rüfenacht, M. Schönherr, and G. Watt, LHAPDF6: Parton density access in the LHC precision era, Eur. Phys. J. C 75, 132 (2015). 